\title{
Hemogasometria e ânion gap em equinos tratados com soluções eletrolíticas enterais contendo diferentes fontes de energia
}

\author{
Blood gas analysis and anion gap in horses treated with enteral electrolyte solutions containing \\ different energy sources
}

\author{
Sheila Kreutzfeld de Farias ${ }^{\text {** }}$ José Dantas Ribeiro Filho ${ }^{\mathrm{I}}$ Athina Chaves Donner ${ }^{\mathrm{I}}$ \\ Waleska de Melo Ferreira Dantas ${ }^{\mathrm{I}}$ Cláudio Luís Nina Gomes ${ }^{\mathrm{I}}$
}

\section{RESUMO}

\begin{abstract}
No presente estudo, foram comparados os efeitos de soluções eletroliticas contendo diferentes fontes de energia administradas via enteral por sonda naso-esofágica de pequeno calibre em fluxo contínuo sobre o equilíbrio ácido base em equinos. Foram utilizadas seis fêmeas adultas em dois quadrados latinos $6 \times 3$ simultâneos em modelo misto. Os animais foram distribuídos em três grupos e submetidos a cada um dos seguintes tratamentos: SEDext $-5 \mathrm{~g}$ de cloreto de sódio, $0,5 \mathrm{~g}$ de cloreto de potássio, $0,2 \mathrm{~g}$ de pidolato de magnésio, $1 \mathrm{~g}$ de gluconato de cálcio e $10 \mathrm{~g}$ de dextrose diluídos em $1.000 \mathrm{~mL}$ de água. Osmolaridade mensurada $228 \mathrm{mOsmol}$ $L^{-1}$; SEMalt - $5 \mathrm{~g}$ de cloreto de sódio, $0,5 \mathrm{~g}$ de cloreto de potássio, $0,2 \mathrm{~g}$ de pidolato de magnésio, $1 \mathrm{~g}$ de gluconato de cálcio $e$ $10 \mathrm{~g}$ de maltodextrina diluídos em $1.000 \mathrm{~mL}$ de água. Osmolaridade mensurada: $181 \mathrm{mOsmol} L^{-1}$ e SEProp - $5 \mathrm{~g}$ de cloreto de sódio, $0,5 \mathrm{~g}$ de cloreto de potássio, $0,2 \mathrm{~g}$ de pidolato de magnésio e $10 \mathrm{~g}$ de propionato de cálcio diluídos em $1.000 \mathrm{~mL}$ de água. Osmolaridade mensurada: $282 \mathrm{mOsm} L^{-1}$. As soluções eletrolíticas foram administradas na dose de $15 \mathrm{~mL} \mathrm{~kg}^{-1} \mathrm{~h}^{-1}$, durante 12 horas. Os tratamentos com soluções eletrolíticas enterais contendo dextrose, maltodextrina ou propionato de cálcio não alteraram os valores da hemogasometria.
\end{abstract}

Palavras-chave: equilíbrio ácido base, hidratação enteral, dextrose, maltodextrina, propionato de cálcio.

\section{ABSTRACT}

The present study compared the effects of electrolyte solutions containing different sources of energy that were administrated through enteral route by naso-esophageal probe of small-caliber with continuous flow on the acid base balance in horses. Six adult females were used in two simultaneous $6 \times 3$ latin squares mixed model. The animals were divided into three groups and received the following treatments: SEDext $-5 \mathrm{~g}$ of sodium chloride, $0.5 \mathrm{~g}$ of potassium chloride, $0.2 \mathrm{~g}$ of magnesium pidolate, $1 \mathrm{~g}$ of calcium gluconate and $10 \mathrm{~g}$ of dextrose diluted in $1.000 \mathrm{~mL}$ of water. The osmolality measured


of potassium chloride, $0.2 \mathrm{~g}$ of magnesium pidolate, $1 \mathrm{~g}$ of calcium gluconate and $10 \mathrm{~g}$ of maltodextrin diluted in $1.000 \mathrm{~mL}$ of water. The osmolality measured was of $181 \mathrm{mOsmol} \mathrm{L^{-1 }}$ and SEProp - $5 \mathrm{~g}$ of sodium chloride, $0.5 \mathrm{~g}$ of potassium chloride, $0.2 \mathrm{~g}$ of magnesium pidolate, $1 \mathrm{~g}$ of calcium gluconate and $10 \mathrm{~g}$ of calcium propionate diluted in $1.000 \mathrm{~mL}$ of water. The osmolality measured was of $282 \mathrm{mOsm} \mathrm{L}^{-1}$. The electrolyte

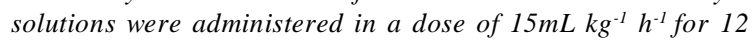
hours. The treatments with enteral electrolyte solutions containing dextrose, maltodextrin and calcium propionate did not change blood gas analysis values in these animals.

Key words: acid base balance, enteral fluidtherapy, dextrose, maltodextrin, calcium propionate.

\section{INTRODUÇÃO}

Nos equinos, alterações hidro-eletrolíticas e ácido base usualmente aparecem associadas a importantes doenças ou síndromes como diarreia, cólica, peritonite, choque endotoxêmico, duodenojejunite proximal, insuficiência renal, exercício físico extenuante, entre outros. Já, na rotina clínica, a correção desses distúrbios é comumente realizada pela administração de soluções eletrolíticas por via enteral ou intravenosa (RIBEIRO FILHO et al., 2007).

'Departamento de Veterinária, Universidade Federal de Viçosa (UFV), 36570-000, Viçosa, MG, Brasil. E-mail: sheilakfarias@yahoo.com.br. *Autor para correspondência. 
Algumas vezes, as enfermidades determinam o aparecimento de inapetência ou anorexia, ou, em alguns casos, o paciente necessita permanecer sem ingerir alimento por um determinado período, o que pode gerar o aparecimento de hipoglicemia. Nesses casos, torna-se necessário acrescentar às soluções eletrolíticas substâncias precursoras de energia. A hidratação enteral, pode ser instituída nos equinos por meio da via nasogástrica, com soluções preparadas para repor a necessidade individual do paciente, podendo ser considerada, atualmente, a opção de eleição em razão de sua eficácia, praticidade e principalmente pelo custo reduzido, tornando-se um importante recurso terapêutico na medicina equina.

A escolha criteriosa da solução adequada para cada tipo de desordem é uma conduta importante e, se feita de forma inapropriada, pode agravar o quadro clínico do animal (RIBEIRO FILHO et al., 2008). Por esse motivo, quando se desenvolve uma solução eletrolítica, é imprescindível avaliar e quantificar sua ação, buscando-se caracterizar os efeitos desejáveis e os adversos.

A hemogasometria e a mensuração de eletrólitos são exames laboratoriais importantes para caracterização e avaliação da intensidade dos desequilíbrios hidro-eletrolíticos e ácido base (RIBEIRO FILHO et al., 2007); por isso, a execução desses exames é necessária nos estudos experimentais com hidratação para demonstrar os efeitos de soluções eletrolíticas sobre a homeostase dos animais.

Os estudos em equinos utilizando soluções eletrolíticas enterais contendo substâncias precursoras de energia ainda são escassos e, até o momento, não responderam as principais questões como, por exemplo: quais as substâncias precursoras de energia que podem ser administradas por essa via? Elas podem ocasionar acidose como acontece nos ruminantes? Qual a dose? Há substâncias que podem substituir a glicose nessa espécie? O presente estudo objetivou avaliar o efeito de soluções eletrolíticas contendo diferentes fontes de energia, administradas por via enteral em fluxo contínuo durante 12 horas sobre o equilíbrio ácido base em equinos.

\section{MATERIAL E MÉTODOS}

Utilizaram-se seis equinos hígidos, fêmeas (não gestantes), sem raça definida, com idade média de dois anos, bom escore corporal (SPEIRS, 1997) e peso corporal médio de $258 \mathrm{~kg}$. Uma semana antes do experimento, após avaliação clínica, foi feito o controle de ectoparasitas (deltametrina a 0,025\%) e endoparasitas (praziquantel + ivermectina). Os equinos foram alojados em baias individuais para adaptação à dieta com água, feno de coast cross (Cynodon dactylon) à vontade, ração comercial concentrada duas vezes ao dia, em quantidade equivalente a $1 \%$ do peso corporal e suplemento mineral, $50 \mathrm{~g}$ ao dia.

Os animais foram distribuídos aleatoriamente em três grupos, cada um contendo seis animais (Tabela 1). Foi realizado cross over 6x3 (seis animais $\mathrm{x}$ três tratamentos). $\mathrm{O}$ intervalo entre os períodos de tratamento foi de sete dias. Os tratamentos foram assim constituídos: o grupo SEDext foi tratado com solução eletrolítica contendo $5 \mathrm{~g}$ de $\mathrm{NaCl}^{\mathrm{a}}, 0,5 \mathrm{~g}$ de $\mathrm{KCl}^{\mathrm{b}}, 0,2 \mathrm{~g}$ de pidolato de $\mathrm{Mg}^{\mathrm{c}}, 1 \mathrm{~g}$ de gluconato de cálcio ${ }^{\mathrm{d}}$ e $10 \mathrm{~g}$ de dextrose ${ }^{\mathrm{e}}$ diluídos em $1.000 \mathrm{~mL}$ de água (osmolaridade mensurada: $228 \mathrm{mMol} \mathrm{L}^{-1}$ ); o grupo SEMalt recebeu solução eletrolítica contendo $5 \mathrm{~g}$ de $\mathrm{NaCl}, 0,5 \mathrm{~g}$ de $\mathrm{KCl}, 0$,2g de pidolato de magnésio, $1 \mathrm{~g}$ de gluconato de cálcio e $10 \mathrm{~g}$ de maltodextrina ${ }^{\mathrm{f}}$ diluídos em 1.000mL de água (osmolaridade mensurada: $181 \mathrm{mMol} \mathrm{L}^{-1}$ ) e; no grupo SEProp, foi administrada solução eletrolítica composta por $5 \mathrm{~g}$ de $\mathrm{NaCl}, 0,5 \mathrm{~g}$ de $\mathrm{KCl}, 0,2 \mathrm{~g}$ de pidolato de magnésio e $10 \mathrm{~g}$ de propionato de cálcio ${ }^{g}$ diluídos em $1.000 \mathrm{~mL}$ de água (osmolaridade mensurada: $282 \mathrm{mMol} \mathrm{L}^{-1}$ ). As soluções eletrolíticas enterais foram administradas na dose de $15 \mathrm{~mL} \mathrm{~kg}^{-1} \mathrm{~h}^{-1}$ em fluxo contínuo por sonda naso-esofágica de pequeno calibre.

Amostras de sangue foram coletadas anaerobicamente para análise hemogasométrica, por meio de punção da veia jugular, em seringas plásticas descartáveis de $3 \mathrm{~mL}$, previamente heparinizadas e acondicionadas em água com gelo, sendo encaminhadas imediatamente para análise em gasômetro. Foram mensuradas as seguintes variáveis: $\mathrm{pH}_{(\mathrm{v})}$; pressão parcial de oxigênio - $\mathrm{pO}_{2(\mathrm{v})}$; pressão parcial do dióxido de carbono - $\mathrm{pCO}_{2(\mathrm{v})}$; concentração total do dióxido de carbono - $\mathrm{tCO}_{2(\mathrm{v})}$; concentração de

Tabela 1 - Distribuição dos animais nos tratamentos.

\begin{tabular}{|c|c|c|}
\hline Período & Animais & Tratamentos \\
\hline $1^{\circ}$ & 1 e 2 & SEDext \\
\hline $1^{\mathrm{o}}$ & 3 e 4 & SEMalt \\
\hline $1^{\mathrm{o}}$ & 5 e 6 & SEProp \\
\hline \multicolumn{3}{|c|}{ Sete dias de intervalo } \\
\hline $2^{\underline{o}}$ & 5 e 6 & SEDext \\
\hline $2^{\underline{o}}$ & 1 e 2 & SEMalt \\
\hline $2^{-}$ & 3 e 4 & SEProp \\
\hline \multicolumn{3}{|c|}{ Sete dias de intervalo } \\
\hline $3^{\mathrm{o}}$ & 3 e 4 & SEDext \\
\hline $3^{\circ}$ & 5 e 6 & SEMalt \\
\hline $3^{\circ}$ & 1 e 2 & SEProp \\
\hline
\end{tabular}


bicarbonato - $\mathrm{cHCO}_{3(\mathrm{v})}^{-}$; concentração de base titulável - cBase $_{(\mathrm{v})}$ e saturação de oxigênio $-\mathrm{sO}_{2(\mathrm{v})}$. O valor dos ânions gap $(\mathrm{AG})$ foi obtido adotando-se a fórmula: $\mathrm{AG}$ $\left(\mathrm{mEq} \mathrm{L}^{-1}\right)=\left(\mathrm{Na}^{+}+\mathrm{K}^{+}\right)-\left(\mathrm{Cl}^{-}+\mathrm{HCO}_{3}^{-}\right)$. As análises foram realizadas nos intervalos: TOh (imediatamente antes da hidratação), T6h (seis horas de hidratação), T12h (término da hidratação) e T24h (doze horas após o término da hidratação).

Para avaliação do efeito dos tratamentos, foi utilizada análise de variância baseada em planejamento de medidas repetidas, ou seja, foi avaliado cada tratamento em vários tempos de acompanhamento. Foi analisada também a influência do tempo e a interação entre o tratamento e o tempo. Quando a análise foi significativa para um ou mais fatores, foi utilizado o teste de Tukey (SAEG, 2007). Todos os resultados foram considerandos significativos quando $\mathrm{P}<0,05$.

\section{RESULTADOS E DISCUSSÃO}

Os resultados dos valores do $\mathrm{pH}_{(\mathrm{v})}, \mathrm{pO}_{2(\mathrm{v})}$, $\mathrm{pCO}_{2(\mathrm{v})}, \mathrm{cHCO}_{3(\mathrm{v})}^{-}, \mathrm{ctCO}_{2(\mathrm{v})}, \mathrm{cBase}_{(\mathrm{v})}, \mathrm{sO}_{2(\mathrm{v})}$ e Ânion gap com as médias e desvios padrão estão expressos nas tabelas 2 e 3. Na avaliação da $\mathrm{pO}_{2(\mathrm{v})}, \mathrm{tCO}_{2(\mathrm{v})}, \mathrm{cHCO}_{3(\mathrm{v})}^{-}$, $\mathrm{sO}_{2(\mathrm{v})}$ e ânion gap, não foram encontradas diferenças na comparação entre tratamentos, nem entre os tempos dentro de cada tratamento $(\mathrm{P}>0,05)$. Além disso, à exceção do Ânion gap, os valores dos parâmetros acima citados mantiveram-se na faixa de referência (KANEKO et al., 2008). Comparando o efeito de soluções eletrolíticas enterais e água sobre o equilíbrio ácido base em equinos, SOSA LEÓN et al. (1995) também não detectaram alterações significativas na concentração do bicarbonato, o que torna os resultados da presente pesquisa semelhantes aos obtidos pelos referidos autores, apesar da diferença da composição e do volume das soluções utilizadas em ambos os estudos. Por sua

Tabela 2 - Médias e desvios padrão do $\mathrm{pH}_{(\mathrm{v})}, \mathrm{pO}_{2(\mathrm{v})}(\mathrm{mmHg}), \mathrm{pCO}_{2(\mathrm{v})}(\mathrm{mmHg}), \mathrm{ctCO}_{2(\mathrm{v})}\left(\mathrm{mmol} \mathrm{L}^{-1}\right), \mathrm{cHCO}_{3(\mathrm{v})}^{-}\left(\mathrm{mmol} \mathrm{L}^{-1}\right) \mathrm{e} \mathrm{cBase}_{(\mathrm{v})}\left(\mathrm{mmol} \mathrm{L}^{-1}\right)$ de equinos tratados com soluções eletrolíticas para uso enteral contendo dextrose (SEDext), maltodextrina (SEMalt) ou propionato de cálcio (SEProp), administradas por sonda nasogástrica de pequeno calibre e fluxo contínuo $\left(15 \mathrm{~mL} \mathrm{~kg}^{-1} 12 \mathrm{~h}^{-1}\right)$.

\begin{tabular}{|c|c|c|c|c|}
\hline Tratamentos & $\mathrm{T} 0 \mathrm{~h}$ & T6h & $\mathrm{T} 12 \mathrm{~h}$ & $\mathrm{~T} 24 \mathrm{~h}$ \\
\hline \multicolumn{5}{|c|}{$\mathrm{pH}_{(\mathrm{v})}$ (média \pm desvio padrão) } \\
\hline SEDext & $7,39 \pm 0,02^{\mathrm{Aa}}$ & $7,38 \pm 0,01^{\mathrm{Aa}}$ & $7,40 \pm 0,01^{\mathrm{Ba}}$ & $7,41 \pm 0,01^{\mathrm{Aa}}$ \\
\hline SEMalt & $7,40 \pm 0,01^{\mathrm{Aa}}$ & $7,38 \pm 0,03^{\mathrm{Aa}}$ & $7,40 \pm 0,01^{\mathrm{Ba}}$ & $7,40 \pm 0,02^{\mathrm{Aa}}$ \\
\hline SEProp & $7,41 \pm 0,01^{\mathrm{Aab}}$ & $7,39 \pm 0,01^{\mathrm{Ab}}$ & $7,43 \pm 0,01^{\mathrm{Aa}}$ & $7,42 \pm 0,01^{\mathrm{Aa}}$ \\
\hline \multicolumn{5}{|c|}{$\mathrm{pO}_{2(\mathrm{v})}($ média \pm desvio padrão) } \\
\hline SEDext & $39,47 \pm 3,44^{\mathrm{Aa}}$ & $43,03 \pm 5,02^{\mathrm{Aa}}$ & $43,57 \pm 2,83^{\mathrm{Aa}}$ & $39,23 \pm 4,42^{\mathrm{Aa}}$ \\
\hline SEMalt & $42,42 \pm 6,37^{\mathrm{Aa}}$ & $44,02 \pm 4,61^{\mathrm{Aa}}$ & $43,42 \pm 2,62^{\mathrm{Aa}}$ & $40,03 \pm 4,67^{\mathrm{Aa}}$ \\
\hline SEProp & $41,18 \pm 6,09^{\mathrm{Aa}}$ & $38,48 \pm 5,03^{\mathrm{Aa}}$ & $39,7 \pm 6,9^{\mathrm{Aa}}$ & $39,37 \pm 4,08^{\mathrm{Aa}}$ \\
\hline \multicolumn{5}{|c|}{$\mathrm{pCO}_{2(\mathrm{v})}$ (média \pm desvio padrão) } \\
\hline SEDext & $44,66 \pm 1,35^{\mathrm{Aa}}$ & $42,78 \pm 1,64^{\mathrm{Aab}}$ & $41,57 \pm 2,1^{\mathrm{Ab}}$ & $42,23 \pm 1,7^{\mathrm{Aab}}$ \\
\hline SEMalt & $43,6 \pm 2,2^{\mathrm{Aa}}$ & $42,58 \pm 1,93^{\mathrm{Aa}}$ & $40,98 \pm 1,18^{\mathrm{Aa}}$ & $42,55 \pm 2,19^{\mathrm{Aa}}$ \\
\hline SEProp & $43,2 \pm 1,98^{\mathrm{Aa}}$ & $41,47 \pm 2,92^{\mathrm{Aa}}$ & $40,52 \pm 3,03^{\mathrm{Aa}}$ & $41,98 \pm 2,56^{\mathrm{Aa}}$ \\
\hline \multicolumn{5}{|c|}{$\mathrm{tCO}_{2(\mathrm{v})}($ média \pm desvio padrão) } \\
\hline SEDext & $28,15 \pm 1,73^{\mathrm{Aa}}$ & $26,40 \pm 1,27^{\mathrm{Aa}}$ & $26,32 \pm 1,3^{\mathrm{Aa}}$ & $27,25 \pm 1,14^{\mathrm{Aa}}$ \\
\hline SEMalt & $27,63 \pm 1,41^{\mathrm{Aa}}$ & $25,82 \pm 1,31^{\mathrm{Aa}}$ & $25,88 \pm 1,09^{\mathrm{Aa}}$ & $27,48 \pm 2,05^{\mathrm{Aa}}$ \\
\hline SEProp & $27,87 \pm 1,29^{\mathrm{Aa}}$ & $25,93 \pm 1,64^{\mathrm{Aa}}$ & $27,33 \pm 1,59^{\mathrm{Aa}}$ & $28,15 \pm 1,88^{\mathrm{Aa}}$ \\
\hline \multicolumn{5}{|c|}{$\mathrm{cHCO}_{3(\mathrm{v})}^{-}($média \pm desvio padrão $)$} \\
\hline SEDext & $26,78 \pm 1,7^{\mathrm{Aa}}$ & $25,08 \pm 1,26^{\mathrm{Aa}}$ & $25,05 \pm 1,26^{\mathrm{Aa}}$ & $25,93 \pm 1,08^{\mathrm{Aa}}$ \\
\hline SEMalt & $26,32 \pm 1,36^{\mathrm{Aa}}$ & $24,50 \pm 1,3^{\mathrm{Aa}}$ & $24,63 \pm 1,06^{\mathrm{Aa}}$ & $26,18 \pm 2,02^{\mathrm{Aa}}$ \\
\hline SEProp & $26,57 \pm 1,2^{\mathrm{Aa}}$ & $24,63 \pm 1,61^{\mathrm{Aa}}$ & $26,08 \pm 1,52^{\mathrm{Aa}}$ & $26,85 \pm 1,81^{\mathrm{Aa}}$ \\
\hline \multicolumn{5}{|c|}{$\operatorname{cBase}_{(\mathrm{v})}($ média \pm desvio padrão $)$} \\
\hline SEDext & $1,60 \pm 1,74^{\mathrm{Aa}}$ & $-0,02 \pm 1,23^{\mathrm{Aa}}$ & $0,22 \pm 1,07^{\mathrm{ABa}}$ & $1,08 \pm 1,1^{\mathrm{Aa}}$ \\
\hline SEMalt & $1,27 \pm 1,25^{\mathrm{Aa}}$ & $-0,70 \pm 1,53^{\mathrm{Aa}}$ & $-0,22 \pm 1,09^{\mathrm{Ba}}$ & $1,28 \pm 1,97^{\mathrm{Aa}}$ \\
\hline SEProp & $1,58 \pm 1,16^{\mathrm{Aab}}$ & $-0,25 \pm 1,34^{\mathrm{Ab}}$ & $1,63 \pm 1,12^{\mathrm{Aab}}$ & $2,22 \pm 1,63^{\mathrm{Aa}}$ \\
\hline
\end{tabular}

Análise de variância (medidas repetidas).

As médias na mesma coluna, seguidas por letras maiúsculas diferentes, e as médias na mesma linha, seguidas por letras minúsculas diferentes, diferem pelo teste de Tukey $(\mathrm{P}<0,05)$.

T0h (imediatamente antes do início da hidratação), T6h (seis horas de hidratação), T12h (12 horas de hidratação) e T24h (12 horas após o término da hidratação).

Ciência Rural, v.41, n.9, set, 2011. 
Tabela 3 - Médias e desvios padrão da $\mathrm{sO}_{2(\mathrm{v})}(\%)$, Ânion $\mathrm{Gap}\left(\mathrm{mmol} \mathrm{L}^{-1}\right)$ e cloreto $\left(\mathrm{mmol} \mathrm{L}^{-1}\right)$ de equinos tratados com soluções eletrolíticas para uso enteral contendo dextrose (SEDext), maltodextrina (SEMalt) ou propionato de cálcio (SEProp), administradas por sonda nasogástrica de pequeno calibre e fluxo contínuo $\left(15 \mathrm{~mL} \mathrm{~kg}^{-1} 12 \mathrm{~h}^{-1}\right)$.

\begin{tabular}{lcccc}
\hline Tratamentos & T0h & T6h & T12h & T24h \\
\hline & & $\mathrm{sO}_{2(v)}($ média \pm desvio padrão $)$ & \\
SEDext & $73,83 \pm 4,42^{\mathrm{Aa}}$ & $77,18 \pm 5,56^{\mathrm{Aa}}$ & $79,02 \pm 2,78^{\mathrm{Aa}}$ & $73,77 \pm 7,20^{\mathrm{Aa}}$ \\
SEMalt & $75,22 \pm 4,40^{\mathrm{Aa}}$ & $76,03 \pm 2,84^{\mathrm{Aa}}$ & $78,10 \pm 3,08^{\mathrm{Aa}}$ & $75,25 \pm 6,97^{\mathrm{Aa}}$ \\
SEProp & $75,92 \pm 7,40^{\mathrm{Aa}}$ & $71,40 \pm 7,40^{\mathrm{Aa}}$ & $74,97 \pm 7,23^{\mathrm{Aa}}$ & $75,32 \pm 4,99^{\mathrm{Aa}}$ \\
& & Ânion Gap (média \pm desvio padrão $)$ & \\
SEDext & $29,27 \pm 8,99^{\mathrm{Aa}}$ & $24,12 \pm 6,06^{\mathrm{Aa}}$ & $26,25 \pm 10,44^{\mathrm{Aa}}$ & $20,93 \pm 15,45^{\mathrm{Aa}}$ \\
SEMalt & $25,77 \pm 8,46^{\mathrm{Aa}}$ & $28,98 \pm 13,20^{\mathrm{Aa}}$ & $25,72 \pm 11,64^{\mathrm{Aa}}$ & $19,73 \pm 12,48^{\mathrm{Aa}}$ \\
SEProp & $23,63 \pm 6,51^{\mathrm{Aa}}$ & $26,02 \pm 9,92^{\mathrm{Aa}}$ & $23,33 \pm 11,47^{\mathrm{Aa}}$ & $26,37 \pm 9,72^{\mathrm{Aa}}$ \\
& & Cloreto $($ média \pm desvio padrão $)$ & & \\
SEDext & $90,33 \pm 6,05^{\mathrm{Aa}}$ & $98,5 \pm 4,5^{\mathrm{Aa}}$ & $97 \pm 7,92^{\mathrm{Aa}}$ & $95,33 \pm 10,25^{\mathrm{Aa}}$ \\
SEMalt & $93,83 \pm 6,67^{\mathrm{Aa}}$ & $92,66 \pm 12,67^{\mathrm{Aa}}$ & $93,5 \pm 12,52^{\mathrm{Aa}}$ & $95,5 \pm 12,66^{\mathrm{Aa}}$ \\
SEProp & $95,83 \pm 6,43^{\mathrm{Aa}}$ & $96,17 \pm 8,49^{\mathrm{Aa}}$ & $97,17 \pm 11,84^{\mathrm{Aa}}$ & $89,83 \pm 8,66^{\mathrm{Aa}}$ \\
\hline
\end{tabular}

Análise de variância (medidas repetidas).

As médias na mesma coluna, seguidas por letras maiúsculas diferentes, e as médias na mesma linha, seguidas por letras minúsculas diferentes, diferem pelo teste de Tukey $(\mathrm{P}<0,05)$.

T0h (imediatamente antes do início da hidratação), T6h (seis horas de hidratação), T12h (12 horas de hidratação) e T24h (12 horas após o término da hidratação).

vez, GOMES (2010) relatou diminuição significativa na $\mathrm{ctCO}_{2}$ e $\mathrm{cHCO}_{3}^{-}$em equinos após a administração de solução eletrolítica enteral, convém ressaltar que a composição da solução eletrolítica também foi diferente da utilizada na presente pesquisa. Segundo o referido autor, esse decréscimo foi decorrente da ação acidificante da solução eletrolítica.

Os valores de referência do ânion gap para a espécie equina variam entre 5 e $16,2 \mathrm{mEq} \mathrm{L}^{-1}$ (WHITEHAIR et al., 1995; CARLSON, 1997). Ele é obtido pela diferença entre cátions e ânions $\left(\mathrm{Na}^{+}+\mathrm{K}^{+}\right)-\left(\mathrm{Cl}^{-}+\right.$ $\mathrm{HCO}_{3}^{-}$), é utilizado primariamente para identificar acidose metabólica e confirmar os distúrbios mistos, sendo também útil para o prognóstico de pacientes enfermos (GOSSETT et al., 1987). Índices superiores à faixa de referência indicam acidose metabólica decorrente do aumento dos ânions não mensurados, principalmente o D-lactato, como ocorre na acidose láctica, determinando a diminuição do bicarbonato. Entretanto, quando se observa a tabela 2, percebem-se valores do ânion gap acima da faixa de referência a partir de $\mathrm{T} 0 \mathrm{~h}$. Isso ocorreu porque os valores do cloreto (Tabela 3) nos animais do presente ensaio encontramse abaixo do valor de referência (KANEKO et al., 2008), gerando aumento no ânion gap. Mas pode-se afirmar que os animais não apresentavam acidose metabólica, como demonstra a cBase (v) (Tabela 2). Esse resultado nos conduz à avaliação crítica de tabelas com valores de referência que, algumas vezes, não expressam a realidade de uma raça, de uma faixa etária ou de uma determinada região.
O tratamento SEProp apresentou os maiores valores do $\mathrm{pH}_{(v)}$ em relação aos demais $(\mathrm{P}<0,05)$ após 12 horas de hidratação (T12h). Esse discreto aumento provavelmente ocorreu devido à composição da solução eletrolítica, porém esse acréscimo não tem significado clínico, pois os valores mantiveram-se nos limites fisiológicos para equinos, 7,35 a 7,45 (KANEKO et al., 2008). Ao longo da fase experimental, foi também detectado pequeno aumento $(\mathrm{P}<0,05)$ no $\mathrm{pH}_{(\mathrm{v})}$ nos animais do SEProp (T12h e T24h), mas os valores mantiveram-se na faixa de referência, tornando-os também sem significado clínico. Embora trabalhando com bovinos, GOFF \& HORST (1994) também não detectaram alteração nos valores hemogasométricos após a administração de propionato de cálcio.

Discreta diminuição do $\mathrm{pH}_{(v)}$ foi relatada por GOMES (2010) ao utilizar solução eletrolítica enteral contendo maltodextrina em equinos, entretanto, convém ressaltar que a composição da solução eletrolítica utilizada pelo referido autor era diferente da utilizada no presente ensaio.

Durante a fase experimental, não houve diferença significativa da $\mathrm{pCO}_{2}$ entre os tratamentos $(\mathrm{P}>0,05)$, porém observou-se diferença ao longo do tempo no tratamento SEDext $(\mathrm{P}<0,05)$. Ocorreu diminuição nos valores de $\mathrm{pCO}_{2}$ no T12h (Tabela 2) no referido tratamento e, apesar do decréscimo, os valores mantiveram-se na faixa de referência para a espécie equina (KANEKO et al., 2008). Segundo JOHNSON (1995), ocorre diminuição ou aumento nos valores da $\mathrm{pCO}_{2}$ como resposta compensatória do componente 
respiratório à acidose ou alcalose metabólicas, respectivamente. Portanto, como não houve alteração clinicamente significativa no componente metabólico $\left(\mathrm{cBase}_{(\mathrm{v})}\right)$ em todos os tratamentos, o componente respiratório $\left(\mathrm{pCO}_{2}\right)$ também manteve-se sem alteração. GOMES (2010) relatou o decréscimo da $\mathrm{pCO}_{2}$ (alcalose respiratória) em equinos tratados com solução eletrolítica enteral contendo maltodextrina. Segundo o referido autor, o evento ocorreu devido o desencadeamento da acidose metabólica decorrente da composição da solução eletrolítica enteral, diferindo dos resultados do presente ensaio.

Como demonstra a tabela 2, foi detectada

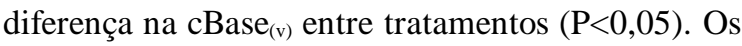
animais do tratamento SEMalt apresentaram valores mais baixos do que o SEProp no tempo $12 \mathrm{~h}$ e, apesar da diferença significativa, a cBase ${ }_{(v)}$ manteve-se na faixa de referência para a espécie equina, -2 a 2 (KANEKO et al., 2008). Na avaliação ao longo do tempo, o tratamento com propionato de cálcio apresentou os menores valores de $\mathrm{cBase}_{(\mathrm{v})}$ no T6h quando comparados com o $\mathrm{T} 24 \mathrm{~h}(\mathrm{P}<0,05)$.

Apesar da ausência de significado clínico, pois a cBase $e_{(v)}$ manteve-se na faixa de normalidade, convém ressaltar que em todos os tratamentos houve decréscimo da cBase $_{(v)}$ no T6h, com subsequente aumento desses valores no T12h. Esse decréscimo no T6h com recuperação no $\mathrm{T} 12 \mathrm{~h}$ pode também ser constatado nos valores do $\mathrm{pH}_{(\mathrm{v})}, \mathrm{pCO}_{2(\mathrm{v})}, \mathrm{tCO}_{2(\mathrm{v})}$ e $\mathrm{HCO}_{3(\mathrm{v})}$, sinalizando que a partir de T6h intensificou-se o mecanismo de compensação, evitando que o decréscimo das referidas variáveis se acentuasse. Normalmente, esse mecanismo de compensação é realizado pelo sistema respiratório $\left(\mathrm{pCO}_{2}\right)$ e pelos rins (urina). Quando se observa o comportamento da $\mathrm{pCO}_{2(\mathrm{v})}$ no T6h e T12h, detecta-se que o mecanismo de compensação via sistema respiratório foi utilizado, ou seja, houve discreta diminuição da $\mathrm{pCO}_{2(v)}$ no T12h.

A propensão à acidificação observada nos animais que receberam os tratamentos SEDext e SEMalt foi decorrente do uso da dextrose e da maltodextrina. Alguns mecanismos hipotéticos podem contribuir para esclarecer o mecanismo de acidificação: o excesso de carboidrato pode ocasionar aumento da glicogenólise, produzindo piruvato em demasia, podendo resultar na síntese de lactato (PROL et al., 2010); açúcares têm tendência a formar ácido após oxidação (STORY et al., 1999) e, segundo ZHANG et al. (2003), açúcares e amido sofrem metabolismo bacteriano no trato gastrintestinal, gerando ácidos orgânicos. Enquanto o SEProp pode ter ocasionado ação sobre a microbiota intestinal, ou seja, o propionato desencadeou aumento no processo fermentativo que por sua vez aumentou os valores do lactato.
A saturação de oxigênio no sangue venoso $\left(\mathrm{sO}_{2 v}\right)$ não apresentou alteração significativa $(\mathrm{P}>0,05)$ durante a hidratação em todos os tratamentos e nos tratamentos ao longo do tempo.

\section{CONCLUSÃO}

A hidratação enteral em equinos tratados com soluções eletrolíticas contendo dextrose (SEDext), maltodextrina (SEMalt) ou propionato de cálcio (SEProp) administradas por sonda nasogástrica de pequeno calibre e fluxo contínuo $\left(15 \mathrm{~mL} \mathrm{~kg}^{-1} 12 \mathrm{~h}^{-1}\right)$ não altera o equilíbrio ácido base em equinos.

\section{FONTES DE AQUISIÇÃO}

a - NaCl PA - Chemco Indústria e Comércio Ltda., Campinas - SP. b - KCl PA - Chemco Indústria e Comércio Ltda., Campinas - SP. c - Pidomag - Laboratório Baldacci S.A., São Paulo - SP.

d - Gluconato de Cálcio - Comércio e Indústria Farmos Ltda., Engenho Novo - RJ.

e - Dextrose - Prolácteos Ind. e Com. Ltda. - Contagem - MG.

f - Maltodextrina - Arve Indústria e Comércio Ltda. - Centev/ UFV, Viçosa - MG.

g - Propionato de cálcio - Comércio e Indústria Farmos Ltda., Engenho Novo - RJ.

\section{COMITÊ DE ÉTICA E BIOSSEGURANÇA}

O delineamento experimental foi submetido ao comitê de ética da Instituição de origem, sendo aprovado sob o protocolo de número 05/2010.

\section{REFERÊNCIAS}

CARLSON, G.P. Fluid, electrolyte, and acid-base balance. In: DIBARTOLA, S.P. Clinical biochemistry of domestic animal. San Diego: Academic, 1997. p.485-516.

GOFF, J.P.; HORST, R.L. Calcium salts for treating hypocalcemia: carrier effects, acid-base balance, and oral versus rectal administration. Journal of Dairy Science, v.77, p.1451-1456, 1994.

GOMES, C.L.N. Efeitos do polietilenoglicol (PEG 3350) e soluções poliônicas administradas por via enteral e intravenosa em equinos. 2010. 130f. Tese (Doutorado em Medicina Veterinária) - Universidade Federal de Viçosa, Viçosa, MG.

GOSSET, K.A. et al. Contribuition of whole blood L-lactate, pyruvate, D-lactate, acetoacetate, and 3-hydroxybutirate concentrations to the plasma anion gap in horses with intestinal disordens. American Journal Veterinary Research, v.48, p.72-75, 1987.

JOHNSON, P.J. Electrolyte and acid-base disturbances in the horses. Veterinary Clinics North America Equine Practice, v.11, p.491-514, 1995.

KANEKO, J.J. et al. Clinical biochemistry of domestic animals. San Diego: Academic, 2008. 932p. 
PROL, A.R. et al. Metabolic acidosis as a complication of intravenous dextrose administration in a patient with insulinoma. Endocrine, v.2, p.143-147, 2010. Disponível em: <http://www.ncbi.nlm.nih.gov/pubmed/20972720b>. Acesso em: 15 out. 2010. doi: 10.1007/s12020-010-9400-y.

RIBEIRO FILHO, J.D. et al. Hemogasometria em equinos com compactação experimental do cólon maior tratados com sene, fluidoterapia enteral e parenteral. Ciência Rural, v.37, p.755-

761, 2007. Disponível em: <http://www.scielo.br/ scielo.php?script=sci_arttext\&pid=S0103-84782007000300024>. Acesso em: 20 de set. 2010. doi: 10.1590/S010384782007000300024 .

RIBEIRO FILHO, J.D. et al. Hemogasometria em cães com desidratação experimental tratados com soluções eletrolíticas comerciais administradas por via intravenosa. Ciência Rural, v.38, p.1914-1919, 2008. Disponível em: <http://www.scielo.br/ s c i e 1 o.p p p ? p i d = S $0 \begin{array}{lllllllllll}0 & 0 & 3 & 4 & 7 & 8 & 2 & 0 & 0 & 8 & 0\end{array}$ $00700017 \&$ script $=$ sci_abstract $\&$ tlng $=$ pt $>$. Acesso em: 19 out. 2010. doi: 10.1590/S0103-84782008000700017.
SAEG - Sistema para análises estatísticas. Versão 9.1. Viçosa: UFV, Fundação Arthur Bernardes, 2007. 301p.

SOSA LEÓN, L.A. et al. The effects of tonicity, glucose concentration e temperature of an oral rehydration solution on its absorption and elimination. Equine Veterinary Journal, Suppl., v.20, p.140-146, 1995.

SPEIRS, V.C. Exame clínico de equinos. Porto Alegre: Artmed, 1997. 286p.

STORY, D.A. et al. The acid-base physiology of crystalloid solutions. Current opinion in critical care, v.5, p.436$439,1999$.

WHITEHAIR, K.J. et al. Clinical applications on quantitative acid-base chemistry. Journal Veterinary Internal Medicine, v.9, p.1-11, 1995.

ZHANG, D.L. et al. D-lactic acidosis secondary to short bowel syndrome. Post Graduate Medicine Journal, v.79, p.110112, 2003. Disponível em: < http://pmj.bmj.com/content/79/ 928/110.full>. Acesso em: 30 set. 2010. doi:10.1136/ pmj.79.928.110. 